\title{
Spatially localized measurement of thermal conductivity using a hybrid photothermal technique
}

Zilong Hua, Heng Ban, Marat Khafizov, Robert Schley, Rory Kennedy, and David H. Hurley’

Citation: Journal of Applied Physics 111, 103505 (2012); doi: 10.1063/1.4716474

View online: http://dx.doi.org/10.1063/1.4716474

View Table of Contents: http://aip.scitation.org/toc/jap/111/10

Published by the American Institute of Physics

\section{Articles you may be interested in}

Parametric study of the frequency-domain thermoreflectance technique

Journal of Applied Physics 112, 103105 (2012); 10.1063/1.4761977

Flash Method of Determining Thermal Diffusivity, Heat Capacity, and Thermal Conductivity

Journal of Applied Physics 32, 1679 (2004); 10.1063/1.1728417

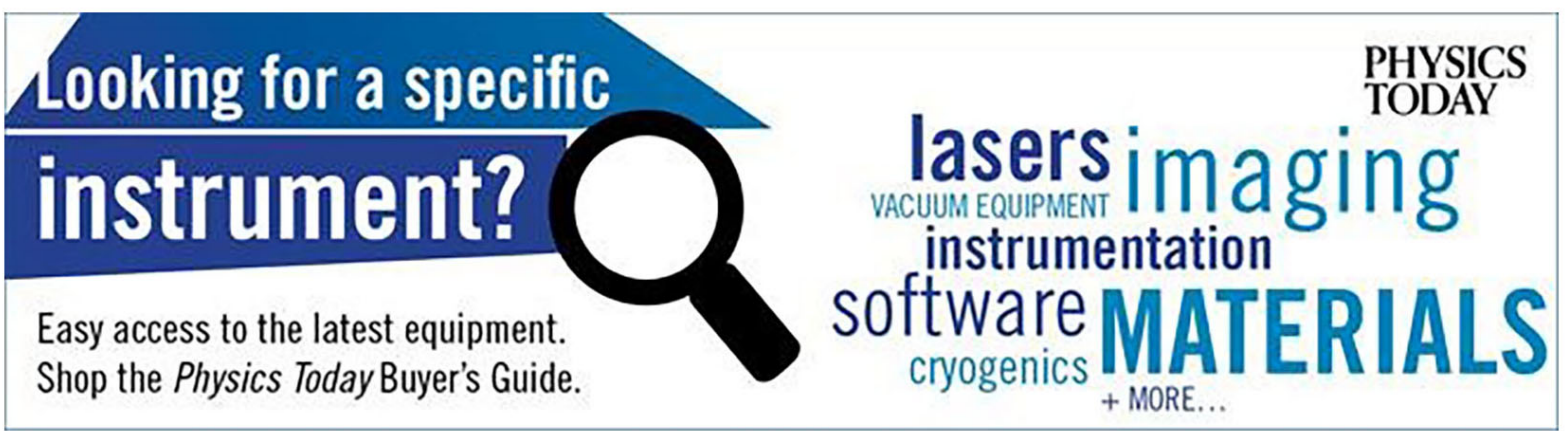




\title{
Spatially localized measurement of thermal conductivity using a hybrid photothermal technique
}

\author{
Zilong Hua, ${ }^{1,2}$ Heng Ban, ${ }^{1}$ Marat Khafizov, ${ }^{2}$ Robert Schley, ${ }^{2}$ Rory Kennedy, ${ }^{2}$ \\ and David H. Hurley ${ }^{2, a)}$ \\ ${ }^{1}$ Mechanical Engineering Department, Utah State University, Logan, Utah 84322-4130, USA \\ ${ }^{2}$ Materials Science and Engineering Department, Idaho National Laboratory, Idaho Falls, Idaho 83415-2209, USA
}

(Received 12 January 2012; accepted 14 April 2012; published online 17 May 2012)

\begin{abstract}
A photothermal technique capable of measuring thermal conductivity with micrometer lateral resolution is presented. This technique involves measuring separately the thermal diffusivity, $D$, and thermal effusivity, $e$, to extract the thermal conductivity, $k=\left(e^{2} / D\right)^{1 / 2}$. To generalize this approach, sensitivity analysis is conducted for materials having a range of thermal conductivities. Application to nuclear fuel is consider by performing experimental validation using two materials $\left(\mathrm{CaF}_{2}\right.$ and $\left.\mathrm{SiO}_{2}\right)$ having thermal properties representative of fresh and high burnup nuclear fuel. The measured conductivities compare favorably with literature values. (C) 2012 American Institute of Physics. [http://dx.doi.org/10.1063/1.4716474]
\end{abstract}

\section{INTRODUCTION}

In nuclear fuel, thermal conductivity, $k$, is intimately related to energy conversion efficiency as well as reactor safety and is arguably one of the most important material properties. It is well known that thermal conductivity of nuclear fuel degrades due to changes in material microstructure brought about by neutron irradiation. Furthermore, the character of the microstructure depends strongly on the local environment and can change drastically over a few millimeters from the fuel element center to the fuel element rim. ${ }^{1}$

Much of the previous work on thermal conductivity of nuclear fuel has concentrated on measuring the combined thermal conductivity of the fuel, cladding, and gap., Whereas these investigations have provided important engineering information, they lack the spatial resolution to address more fundamental aspect of thermal transport. Recently, there has been an effort to understand the fundamental role played by irradiation microstructure in limiting thermal transport. ${ }^{1,4}$ Much of this work has focused on separate effects studies using simulated extended burnup fuel. While these studies have provided significant new insight, it is preferable to perform post irradiation investigations on real fuel samples as they are representative of the irradiation and temperature conditions found in reactor. Gauging the influence of microstructure on these samples will necessitate the development of new spatially resolved tools that can accurately measure thermal conductivity on length scales commensurate with microstructure heterogeneity.

Laser-based methods have emerged as a leading candidate for making precise thermal transport measurements due to their non-contact nature and well defined optical coupling conditions. ${ }^{6,7}$ In general, these methods use a pump laser for transient heating of the sample. This can be implemented in the frequency domain using an amplitude modulated continu-

\footnotetext{
a) Author to whom correspondence should be addressed. Electronic mail: david.hurley@inl.gov.
}

ous wave laser $^{8-11}$ or in the time domain using a pulsed laser. ${ }^{12,13}$ Temperature sensing is accomplished by either recording black body radiation (laser flash) or using a probe laser to record small temperature induced changes in optical reflectivity (thermal reflectance). Laser flash methods, designed to measure the thermal properties averaged over the heat pulse propagation distance, have an ultimate spatial resolution in the depth direction $(\sim 1 \mathrm{~mm})$ determined by the minimum sample thickness. ${ }^{14,15}$ Recently, there has been work on the development of a modified laser flash method capable of providing near millimeter lateral resolution. ${ }^{16}$ This work utilized a liquid nitrogen cooled mercury-cadmium-telluride detector used for localized IR detection of blackbody irradiation. In contrast, thermal reflectance methods can measure thermal properties in the lateral direction with near micron resolution as well as the depth direction with near nanometer resolution. ${ }^{17}$ The spatial resolution of these methods depends on the thermal wavelength or the diffusion length, and is ultimately limited by the laser spot size.

Because most laser-based methods measure relative temperature changes, they are limited to measuring some combination of conductivity, density, and specific heat. Typically researchers extract the thermal conductivity from these measurements by performing an additional experiment to measure specific heat. This requires destructively removing a small volume of the test sample to be placed in a calorimeter. For highly radioactive material, this approach raises serious waste management issues.

The work detailed in this manuscript involves the development of a laser-based technique to remotely measure the thermal conductivity of nuclear fuel on a micron length scale. This approach involves using a hybrid frequency/spatial domain thermal reflectance method to measure the thermal effusivity, $e$, and thermal diffusivity, $D$. The thermal conductivity, $k=\left(e^{2} D\right)^{1 / 2}$, is then extracted from these measurements. A key point is that the effusivity and diffusivity measurements are made at the same location on the sample surface allowing for spatially localized measurement of the thermal conductivity. 


\section{THEORY}

Thermal reflectance methods are based on the analysis of thermal waves. ${ }^{8,9}$ In general, a thermal wave is a nonpropagating diffusion wave defined by a complex wavenumber. ${ }^{18}$ In this section, models of thermal waves used for data analysis will be discussed. The sample geometry considered consists of a thin film of thickness $h$ placed on a semi-infinite substrate. The film has two important functions. First, the film introduces a second boundary condition that explicitly contains an expression for the thermal effusivity. Second, the film ensures strong optical absorption, a requirement for optically transparent substrates. These requisites can be met by coating the sample with a metallic film. Additionally, the film material is chosen to have a large thermoreflectance coefficient (i.e., large $\Delta \mathrm{R} / \Delta \mathrm{T}$ ) at the probe laser wavelength. Both the film and substrate are assumed to be thermally isotropic.

The heat source is provided by an amplitude modulated laser and the temperature field is recorded using a probe laser to sense small temperature induced changes in reflectivity. ${ }^{8,9}$ The amplitude and phase of the probe signal are detected using lockin amplification. However, in the remainder of the paper, we will consider only the phase profile of the temperature field. The phase profile provides direct measurement of the thermal diffusion length, while the amplitude profile is more difficult to interpret due to potential variations in reflectivity across the sample. ${ }^{7}$

\section{A. Frequency domain-1D model}

Measurement of the thermal effusivity is a key element in the procedure to extract thermal conductivity. It is thus important to understand how effusivity enters into the model describing heat flow. In general, the temperature field for 1D sinusoidal heat flow can be represented as ${ }^{11,19}$

$$
T_{j}=A_{j} \exp \left(z \sqrt{i \omega / D_{j}}\right)+B_{j} \exp \left(-z \sqrt{i \omega / D_{j}}\right),
$$

where the subscript $j$ either represents the film (f) or the substrate (s) and $\omega=2 \pi f$ is the angular frequency. The undetermined coefficients are obtained by satisfying the boundary conditions. It is assumed that there is no heat flux into the surrounding air at $\mathrm{z}=0$ and the boundary condition between substrate and film is given by

$$
\left.k_{s} \frac{\partial T_{s}}{\partial z}\right|_{z=h}=\left.k_{f} \frac{\partial T_{f}}{\partial z}\right|_{z=h} .
$$

To keep the analysis simple while retaining the salient features of the proposed method, we neglect the influence of interface resistance. An expression explicitly containing $e$ is obtained by substituting the temperature field in Eq. (1) into this boundary condition

$$
\begin{aligned}
& A_{s} e_{s} \exp \left(h \sqrt{i \omega / D_{s}}\right)+B_{s} e_{s} \exp \left(-h \sqrt{i \omega / D_{s}}\right) \\
& =A_{f} e_{f} \exp \left(h \sqrt{i \omega / D_{f}}\right)+B_{f} e_{f} \exp \left(-h \sqrt{i \omega / D_{f}}\right) .
\end{aligned}
$$

If we consider the simple case of surface heating then the phase lag, $\varphi$, of the temperature response to the heat source at the surface of the film is given by ${ }^{20}$

$$
\begin{aligned}
& \varphi=\frac{3}{4} \pi+\tan ^{-1}\left[\frac{\cosh ^{2}(N)[\tanh (N)+\beta]\left[\tanh (N)+\beta^{-1}\right]}{\cos ^{2}(N)\left(\beta-\beta^{-1}\right) \tan (N)}\right], \\
& N=\sqrt{\frac{\omega \tau_{f}}{2}}
\end{aligned}
$$

where $\beta=e_{s} / e_{f}$ is defined as the ratio of thermal effusivity of the substrate to that of the film and $\tau_{f}=h^{2} / D_{\mathrm{f}}$ is the characteristic time for heat diffusion in the film. The thermal wave phase lag versus modulation frequency for different values of $\beta$ is shown in Fig. 1(a). In the limit of zero frequency, the thermal wavelength becomes infinite. In this case, the solution is that of a 1D monolithic half-space with the thermal wave phase lag approaching $45^{\circ}$. Conversely, in the high frequency limit, the thermal wavelength becomes small in relation to film thickness and hence the solution returns to a phase lag of $45^{\circ}$. Also note that for the case of $\beta=1$, the flow of heat is not influenced by the boundary between the film and substrate. In principle, the thermal effusivity of the substrate can be obtained in a straight forward manner using single parameter fitting if the thermal properties of the film are known.

In practice, care must be taken to ensure that the experimental geometry approximates the 1D model. The key length scales are the thermal wavelength, $\lambda=(D / 2 \omega)^{1 / 2}$, and the laser spot size, $a$. As a rule of thumb, 1D behavior is reached when $a / \lambda \gg 1$. There are two important points in this regard. First, the thermal wave amplitude decreases with decreasing wavelength. Second, increasing the spot size requires additional pump power to maintain a constant thermal wave amplitude. Thus, to ensure an adequate signal to noise ratio (SNR), a compromise between the $a / \lambda$ ratio and the signal amplitude must be found.
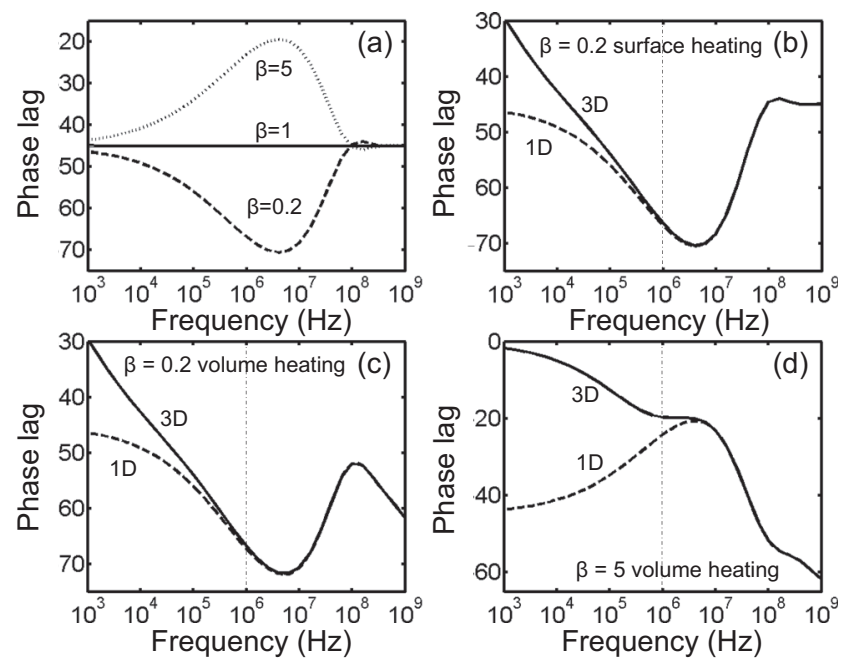

FIG. 1. Calculated thermal wave phase lag versus frequency for $\mathrm{h}=200 \mathrm{~nm}, \mathrm{e}_{\mathrm{f}}=4.9 \mathrm{~kW} \mathrm{~s}{ }^{1 / 2} / \mathrm{m}^{2} \mathrm{~K}, \mathrm{D}_{\mathrm{f}}=5.0 \mathrm{~mm}^{2} / \mathrm{s}$. (a) $1 \mathrm{D}$ model (dashed line $-\beta=0.2$, solid line $-\beta=1$, dotted line $-\beta=5$, corresponding to $\mathrm{e}_{\mathrm{s}}=0.98,4.9$, and $25 \mathrm{~kW} \mathrm{~s}{ }^{1 / 2} / \mathrm{m}^{2} \mathrm{~K}$, respectively). (b) $1 \mathrm{D}$ model (dashed line) and 3D model (solid line) without considering finite optical penetration for $\beta=0.2$. (c) $1 \mathrm{D}$ model (dashed line) and 3D model (solid line) with finite optical penetration for $\beta=0.2$. (d) $1 \mathrm{D}$ model (dashed line) and 3D model (solid line) with finite optical penetration for $\beta=5$. 


\section{B. Frequency domain-3D model}

In order to accurately define the set of experimental parameters for which the 1D model is valid, we consider a full 3D model. The 1D heating source is replaced by a 3D source having a Gaussian radial profile. For a detailed description of this solution, the reader is referred to previous investigations. ${ }^{11,21}$ Here, we present only the results. Figure 1(b) compares the $1 \mathrm{D}$ and $3 \mathrm{D}$ solutions for $\beta=0.2$. At $1 \mathrm{MHz}$, the phase difference of $1 \mathrm{D}$ and $3 \mathrm{D}$ cases is less than $1^{\circ}$ which is comparable to the noise level of the experiment. Analysis reveals that a $0.5^{\circ}$ uncertainty in the phase lag gives rise to approximately a $4 \%$ uncertainty in the substrate effusivity. Again both solutions approach $45^{\circ}$ at high modulation frequencies. The behavior at high frequency is different however if we include volumetric heating with an estimated penetration depth of $\delta=10 \mathrm{~nm}$ for a $400 \mathrm{~nm}$ wavelength beam. This introduces another length scale, namely the optical penetration depth. As a result, the phase lag deviates from $45^{\circ}$ when the thermal wavelength approaches the optical penetration depth as demonstrated in Fig. 1(c). For this comparison, a finite optical penetration is considered for both the 1D and 3D cases. Now to determine the effect of $\beta$ on convergence of the $1 \mathrm{D}$ and $3 \mathrm{D}$ models, we consider the case when the substrate has a thermal effusivity that is much larger than the film (e.g., $\beta=5$ ). This result is shown in Fig. 1(d). For low to moderate frequencies, the phase lag increases with frequency in agreement with the results depicted by the dotted line in Fig. 1(a). The important point here is that the frequency at which the solutions converge increases with increasing $\beta$.

\section{Spatial domain-3D model}

In the spatial scan method, the pump or probe beam is scanned across the sample surface and the phase and amplitude of the thermal wave are recorded. ${ }^{10,22}$ For laterally heterogeneous samples, scanning the pump beam requires solving the governing equations for each pump position. In this case, it is preferable to move the probe beam. Conversely for samples with varying optical reflectivity, it is best to position the probe beam on a highly reflective region of the sample and scan the pump beam. For the current proof of principle study, the sample is laterally homogeneous and uniformly reflective. As a consequence, the probe beam is scanned keeping the pump beam stationary. In general, the thermal wave phase lag, $\Delta \varphi$, is a linear function of scan distance, $\Delta x$, in a region far removed from the pump beam ${ }^{10,25}$

$$
\Delta \varphi=\Delta x \cdot q,
$$

where $q$ is defined as the real part of the thermal wavenumber. For an uncoated single component system, the dispersion relationship has a simple functional form: $q=(\omega / 2 D)^{1 / 2} \cdot{ }^{23}$ For a film/substrate system, the dispersion relationship becomes a complicated function of the thermal properties of the film and substrate. Again we use the same 3D model from Sec. II B but now we vary the separation distance between pump and probe. ${ }^{10,25}$ Model results for the thermal wave phase for a system composed of a thermally conductive film on a thermally
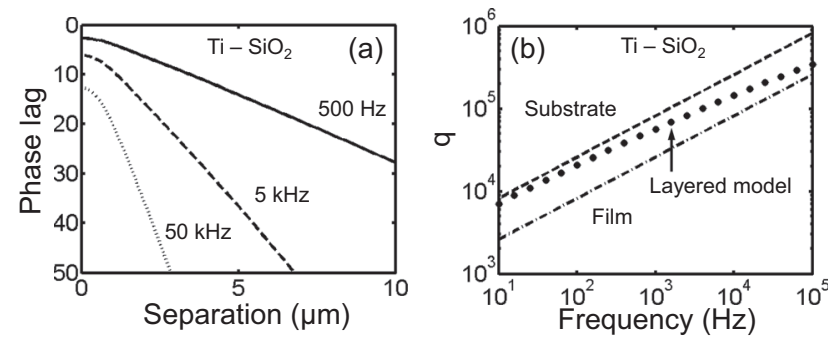

FIG. 2. (a) Calculated thermal wave phase profiles for a two layer system at different frequencies in the spatial domain (solid line- $500 \mathrm{~Hz}$, dashed line $-5 \mathrm{kHz}$, dotted line $-50 \mathrm{kHz}$ ). (b) A $\log -\log$ plot for the thermal wavenumber corresponding to the two layer model (dots). The upper and lower straight lines correspond to a half space composed solely of the substrate and the film materials, respectively. Thermal parameters are listed in Table I under case $\mathrm{I}$.

insulating substrate are shown in the left of Fig. 2. The thermal parameters used in Fig. 2 correspond to case I in Table I. The nonlinear portion of the profile near zero separation reflects the finite beam size of the pump laser.

The right plot in Fig. 2 shows the real part of the thermal wavenumber versus modulation frequency (dots) on a $\log$-log graph. The wavenumber is obtained by measuring the slope, $\Delta \varphi / \Delta \mathrm{x}$, of the phase profile in the far-field of the pump. The upper and lower straight lines corresponds to a half space composed solely of the substrate and film materials, respectively [slope $=1 / 2, y$-intercept $=-1 / 2 \log (2 D)$ ] This figure illustrates that the effective thermal diffusivity transitions from the substrate diffusivity in the low frequency range (a few hundred $\mathrm{Hz}$ ) to the film diffusivity in the high frequency range $(\mathrm{MHz})$. The transition region moves to higher frequencies with increasing substrate diffusivity.

\section{SENSITIVITY ANALYSIS}

To consider the optimal operating range, we make use of the sensitivity $S_{\xi}$ function defined as follows:

$$
S(\xi)=\frac{\partial \varphi}{\partial \ln \xi}=\frac{\varphi(\xi+\Delta \xi)-\varphi(\xi)}{\Delta \xi / \xi},
$$

where $\xi$ is the parameter in question. Four thermal parameters, $k_{s}, k_{f}, D_{s}, e_{s}$, and the heating laser spot size, $a$, are considered as parameters. In this section, we present sensitivity data for two substrate materials representative of a range of nuclear fuel materials. Case I is a low conductivity material representative of high burnup fuel and case II is a moderate conductivity material representative of fresh fuel. The film material, the same for both cases, has thermal properties typical of a thin metallic film. The thermal properties of both substrates and of the film are listed in Table I. Case I represents a system with high thermal contrast and case II represents a system with moderate to low thermal contrast. In Fig. $3($ a), $S(\xi)$ is plotted for a spatial scan at a modulation frequency $50 \mathrm{kHz}$ for case I. This plot illustrates that there is a relatively high sensitivity to $k_{f}$. This behavior is to be contrasted with the results at $100 \mathrm{~Hz}$ shown in Fig. 3(b). For this 
TABLE I. Sample properties for cases I and II.

\begin{tabular}{lccc}
\hline \hline & Sample A & Sample B \\
\hline $\mathrm{k}_{\mathrm{f}}(\mathrm{W} /(\mathrm{m} \mathrm{K}))$ & & 11 \\
$\mathrm{D}_{\mathrm{f}}\left(\mathrm{m}^{2} / \mathrm{s}\right)$ & & $5 \times 10^{-6}$ & \\
$\mathrm{k}_{\mathrm{s}}(\mathrm{W} /(\mathrm{m} \mathrm{K}))$ & 1.1 & & 8.8 \\
$\mathrm{D}_{\mathrm{s}}\left(\mathrm{m}^{2} / \mathrm{s}\right)$ & $5 \times 10^{-7}$ & & $4 \times 10^{-6}$ \\
spot size $(\mu \mathrm{m})$ s.d. & & 1 & \\
spot size $(\mu \mathrm{m})$ f.d. & & 15 & \\
$\mathrm{~h}(\mathrm{~nm})$ & & 200 & \\
\hline
\end{tabular}

case, the sensitivity to $D_{s}$ is larger than the other thermal parameters of the system. This increase in $S\left(D_{S}\right)$ with decreasing frequency is essentially a restatement of the data presented in Fig. 2(b). In Fig. 3(c), $S(\xi)$ is plotted for a spatial scan at a modulation frequency $100 \mathrm{~Hz}$ for case II. Here, in the far field of the pump, the sensitivity to $D_{s}$ is an order of magnitude larger than the sensitivity to other parameters. The sensitivity to the pump beam spot size, $S(a)$, versus scan distance is considered individually in Fig. 3(d). The thermal parameters used for this plot correspond to case II; however, the results can be applied to case I as well. The sensitivity increases rapidly near the origin reflecting the finite spot size of the pump. For large scan distances, there is a constant offset between phase profiles corresponding to different spots sizes and as result $S(a)$ becomes flat with scan distance. Thus, this parameter can be obtained independently from other parameters because it only significantly influences the model results near the origin.
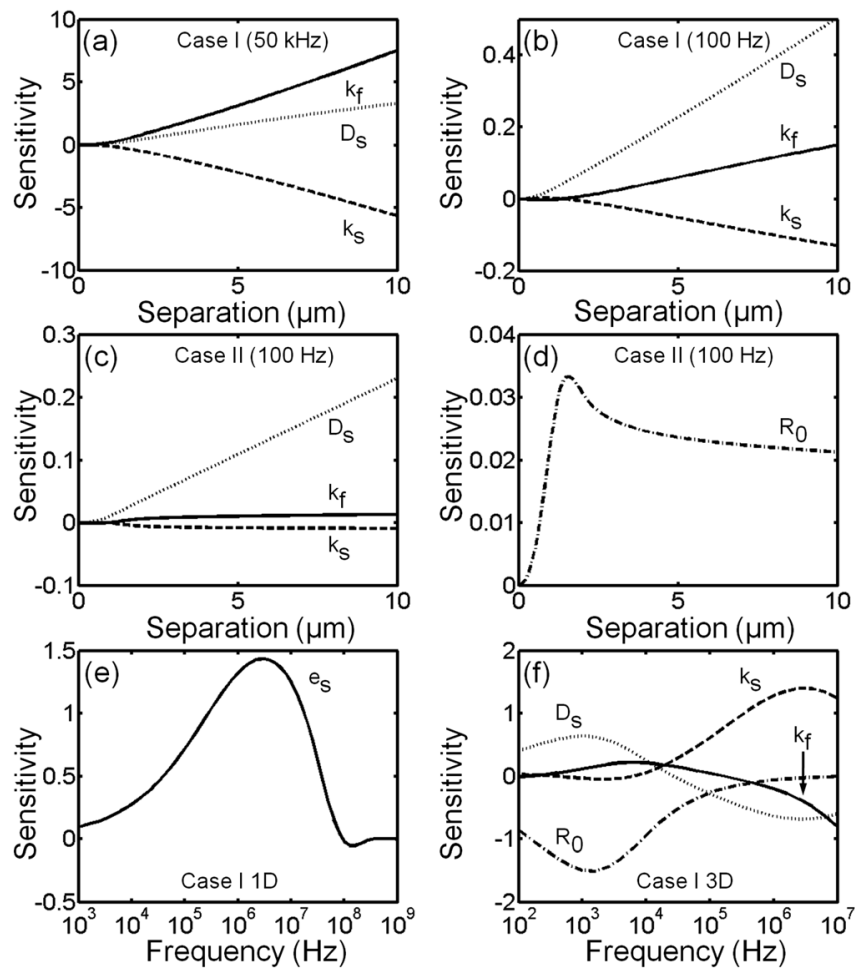

FIG. 3. Sensitivity analysis in the spatial and frequency domain. (a), (b), (c), (d), and (f) Solid-film conductivity, dotted-substrate diffusivity, dashed-substrate conductivity, dashed-dotted-pump spot size. (e) Solidsubstrate effusivity. (a), (b), and (f) Case I. (c), (d), and (e) Case II. Parameters are listed in Table I.
Now we switch to considering sensitivity in the frequency domain. In Fig. 3(e), we plot $S\left(e_{s}\right)$ versus frequency for case I. The sensitivity peaks in the $1-5 \mathrm{MHz}$ range because in this range the thermal wavelength in the film is comparable to the film thickness. Thus, the boundary condition between the film and substrate becomes significant. As noted previously, it is this boundary condition that introduces the thermal effusivity. This result can be generalized to other substrate materials because the thermal properties of the film largely determine the shape $S\left(e_{s}\right)$.

Last, we consider $\mathrm{S}(\xi)$ for a frequency scan with $k_{s}, k_{f}$, $D_{s}$, and $a$ as parameters for case I. Each sensitivity curve displays a peak in the portion of the spectrum shown. Switching to case II pushes these peaks to higher frequency. The important point here is that there is no portion of the spectrum where the sensitivity to any single parameter is dominant. Thus, frequency domain scans typically require multi parameter fitting.

\section{MEASUREMENT PROCEDURE}

In this section, we describe a procedure for measuring thermal conductivity of the substrate. To do this, we need to identify the system parameters that must be measured. As previously stated, both $D_{s}$ and $e_{s}$ must be measured to extract $k_{s}$. In addition, the thin metallic transducer film typically has nanometer size crystallites and as a result the thermal conductivity is substantially different than the bulk due to grain boundary scattering. ${ }^{23}$ Therefore, the thermal transport properties of the film must also be measured. This is accomplished using a baseline sample composed of a substrate with known thermal conductivity and diffusivity. To ensure that both metal films have the same thermal properties (i.e., same crystallite size) and thickness, the baseline sample and sample of interest must be coated simultaneously. Assessing these thermal parameters requires fitting model output to experimental results. This necessitates an accurate measurement of the film thickness, which is accomplished using the picosecond acoustic technique. ${ }^{24}$

Justification for our measurement procedure is found in the sensitivity curves given in Sec. III. Essentially there are three unknown thermal constants that must be measured in order to obtain the substrate conductivity (i.e., $k_{f}, D_{s}$, and $e_{s}$ ). The intent of this section is to provide an approach that is appropriate for a range of substrate materials having thermal properties representative of nuclear fuel. In this regards, we consider substrate materials that are both thermally similar and dissimilar to the metal film. An outline of this approach is given below:

1. Measure the thermal conductivity of the film. A baseline sample with a known substrate material is needed for this task. Figure 3(a) demonstrates that a thermally insulating substrate such as $\mathrm{SiO}_{2}$ is a good choice for the baseline sample due to high sensitivity on $k_{f}$. It is assumed that the specific heat of the film is the same as for the bulk material. For fully dense films, this is a reasonable assumption. Thus, we fit experiment data and theory result using only two fitting parameters ( $a$ and $k_{f}$ ). As mentioned previously, the spot size only influences the phase profile near 
the origin and hence $k_{f}$ is essentially obtained independently of the spot size.

2. Measure the thermal diffusivity of the substrate. Here we plot the real part of the thermal wavenumber versus frequency on a log-log graph. We can envision two scenarios. One: a linear relationship is observed at low frequencies indicating that we are only sensing the substrate (see Fig. 2(b)). In this case, $D_{s}$ can be obtained in a straight forward manner with high confidence. Two: a linear relationship is not reached with decreasing frequency. In this case, $D_{s}$ is substantially lower than $D_{f}{ }^{25}$ and $D_{s}$ must be obtained including $k_{s}$ as an additional parameter. This situation is to be avoided if possible because multiparameter fitting raises issues concerning uniqueness. ${ }^{6,26}$ It is worth noting that in principle a linear relationship can be reached with ever decreasing modulation frequency. However, in real experiments, there is a lower frequency limit determined by system noise.

3. Measure the thermal effusivity of the substrate. Two important points must be considered in this regard. First to ensure peak sensitivity to $e_{s}$, the thermal wavelength in the film must be tuned to be approximately equal to the film thickness. Second, we chose a relatively large pump laser spot size in relation to the optimal thermal wavelength to ensure a $1 \mathrm{D}$ experimental geometry. In the $1 \mathrm{D}$ limit, the phase profile versus frequency can be fit using the substrate effusivity as the sole fitting parameter. After the effusivity is measured, we can go back and compare the $1 \mathrm{D}$ and $3 \mathrm{D}$ theoretic phase profiles using fitted values to ensure that the $1 \mathrm{D}$ condition was met. The thermal conductivity can now be extracted using the measured values for diffusivity and effusivity.

\section{EXPERIMENT AND SAMPLE DESCRIPTION}

The diagram of our experiment setup is shown in Fig. 4. The pump and probe ${ }^{27}$ beams are both derived from continuous wave lasers having wavelengths of $532 \mathrm{~nm}$ and $655 \mathrm{~nm}$,

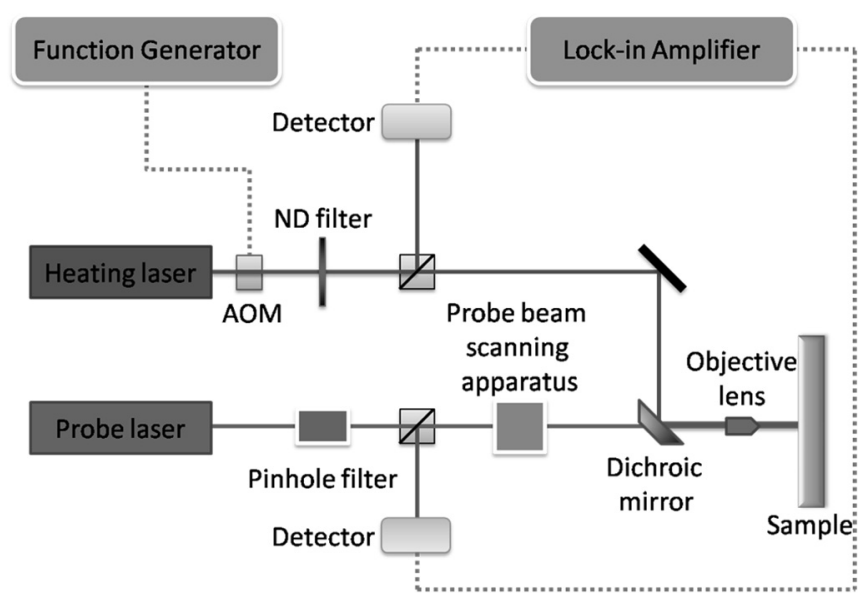

FIG. 4. Experimental setup. An AOM is used for amplitude modulation of the pump beam. Both the pump and probe beam are focused onto the sample using a single microscope objective. The probe is scanned relative to the pump by changing the entrance angle into the objective. respectively. The power of the pump and probe laser beams reaching the sample surface is $\sim 3 \mathrm{~mW}$ and $500 \mu \mathrm{W}$, respectively. An acousto-optic modulator (AOM) is used for amplitude modulation of the pump laser. The upper modulation frequency, $\sim 5 \mathrm{MHz}$, is determined by the reduced modulation depth of the AOM with increasing frequency. Scanning of the probe relative to the pump beam is achieved by sending the probe beam through a confocal lens pair (of focal length $100 \mathrm{~mm}$ ). ${ }^{28}$ The first lens in the confocal system is attached to a stage that allows the lens and the probe beam to be translated in the $x$-y plane. The probe beam propagation vector remains collinear with the lens axis. The second lens converts the $x$-y motion of the beam into a change in entrance angle into the objective. The scan limit using this optical system is approximately $100 \mu \mathrm{m}$. We use a $50 \times$ objective lens for the spatial domain measurements and a $10 \times$ objective lens for the frequency domain measurements. The pump beam is guided along the optical axis of the objective using a dichroic beam splitter placed after the lens pair. The sample surface as well as the pump and probe laser beams are imaged onto a CCD camera to check beam overlap and surface quality. The reflected probe light is collected by a photodiode after passing a wavelength selective absorption filter to block the pump light. The output signal is then sent to a lock-in amplifier.

For the proof of principle experiment, we use two substrate materials: sample A-fused silica $\left(\mathrm{SiO}_{2}\right)$ is a material with thermal properties representative of high burnup nuclear fuel, and sample $\mathrm{B}$-calcium fluoride $\left(\mathrm{CaF}_{2}\right)$ is a material with thermal properties representative of fresh nuclear fuel. Additionally we used the $\mathrm{SiO}_{2}$ as our baseline sample to determine $k_{\mathrm{f}}$. It is noted that our proof of principle experiment and our baseline measurement on $\mathrm{SiO}_{2}$ were performed separately.

Titanium has been identified as the optimal transducer material due to its large thermoreflectance coefficient at the probe laser wavelength. A film thickness of $200 \mathrm{~nm}$ was suggested by sensitivity analysis. Titanium thin films were deposited on $\mathrm{SiO}_{2}$ (Sample A) and $\mathrm{CaF}_{2}$ (sample B) substrates simultaneously using thermal sputtering. Picosecond acoustics was used to obtain the film thickness. The measured film thickness is $170( \pm 4) \mathrm{nm}$.

\section{RESULTS AND DISCUSSION}

Baseline measurements on the $\mathrm{SiO}_{2}$ sample using both the spatial and frequency domain methods are presented in Fig. 5. We use these experimental results to determine $k_{f}$ based on the published values for $\mathrm{SiO}_{2}$ $\left(k_{\mathrm{s}}=1.4 \mathrm{~W} /(\mathrm{m} \mathrm{K})\right.$ and $\left.D_{\mathrm{s}}=9.5 \times 10^{-7} \mathrm{~m}^{2} / \mathrm{s}\right)$. Figure $5(\mathrm{a})$ shows spatial domain phase profiles at $5 \mathrm{kHz}, 10 \mathrm{kHz}$, and $50 \mathrm{kHz}$ and corresponding least square fits using the model discussed in Sec. II C. The best fit value for $k_{f}$ is determined to be $11.0 \mathrm{~W} /(\mathrm{m} \mathrm{K})$ which is substantially lower than the bulk value of $21.9 \mathrm{~W} /(\mathrm{m} \mathrm{K})$. To confirm this measurement, we also performed a fit on the frequency domain data shown in Fig. 5(b). The fitted value in this case $k_{\mathrm{f}}=10.8 \mathrm{~W} /(\mathrm{m} \mathrm{K})$ is in good agreement with spatial domain measurement. 

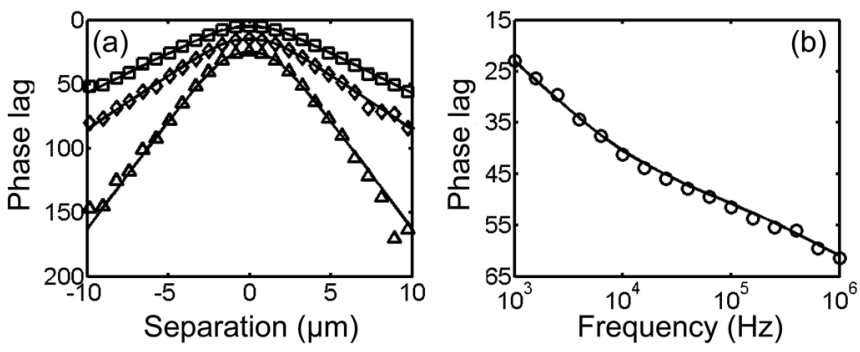

FIG. 5. Thermal conductivity measurement of the film for baseline sample. (a) Spatial domain data (sqaure $-5 \mathrm{kHz}$, diamond $-10 \mathrm{kHz}$, triangle$50 \mathrm{kHz}$ ). Solid lines are best fit model results. (b) Frequency domain (experimental data—circles, solid line-best fit model result).

To extract $D_{s}$ of sample A and sample B we performed spatial domain scans in the frequency range from $20 \mathrm{~Hz}$ to $100 \mathrm{kHz}$. The real part of the thermal wavenumber is obtained from each individual profile using linear fitting. The results are plotted in Fig. 6 on a log-log scale. In order to simplify the fitting process, we started the analysis by looking at the low frequency portion of the data that had a linear relationship with a slope of $1 / 2$. To be consistent with our measurement noise, we use a $\sim 5 \%$ tolerance for the slope. A slope that fell outside this tolerance requires including $k_{\mathrm{s}}$ in the fitting routine. The frequency dependence of the wavenumber of sample A suggests a thermally slow substrate. Accordingly, we only use the low frequency region from $20 \mathrm{~Hz}$ to $100 \mathrm{~Hz}$ to extract $\mathrm{D}_{\mathrm{s}}(\Delta \log (q) / \Delta \log (\omega)=0.475)$. Sample B has a comparable thermal diffusivity to the Ti film as indicated by the linear relationship across the entire frequency range sampled $(\Delta \log$ $(q) / \Delta \log (\omega)=0.498)$. Therefore, in both cases, $D_{s}$ are obtained directly and the results are given in Table II.

To determine $e$, we performed a phase measurement at distinct frequencies and applied the phase lag expression in Eq. (4). From the discussion above, the optimal frequency for the effusivity measurement fell in a narrow window. The bounds of this window are determined by three requirements. First, the thermal wavelength in the film must be comparable to the film thickness. Second, the ratio of the pump spot size to the thermal wavelength needs to be large $(a / \lambda \gg 1)$ to validate our 1D analysis. Third, the SNR must be sufficiently large for accurate measurements. Here, it is important to note that the SNR decreases with increasing frequency due to decreasing thermal wave amplitude and decreasing modu-
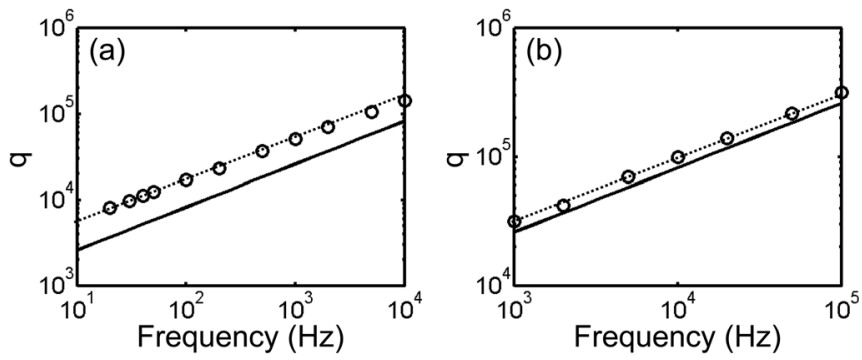

FIG. 6. The log-log plots of the real part of the thermal wavenumber with respect to frequency for samples A (left) and B (right) in the frequency region from $100 \mathrm{~Hz}$ to $100 \mathrm{kHz}$. (Circle-experiment, solid-straight lines associated with $D_{\mathrm{f}}$, dotted-best fit line).
TABLE II. Measured and the literature value of thermal conductivity, diffusivity, and effusivity of unknown samples. ${ }^{32}$

\begin{tabular}{|c|c|c|}
\hline & $\begin{array}{l}\text { Sample A } \\
\left(\mathrm{SiO}_{2}\right)\end{array}$ & $\begin{array}{l}\text { Sample B } \\
\left(\mathrm{CaF}_{2}\right)\end{array}$ \\
\hline Phase lag (deg) & 60.4 & 46.3 \\
\hline Effusivity $\left(\mathrm{J} / \mathrm{m}^{2} \mathrm{~s}^{1 / 2} \mathrm{~K}\right)$, measured & 1490 & 4570 \\
\hline Effusivity $\left(\mathrm{J} / \mathrm{m}^{2} \mathrm{~s}^{1 / 2} \mathrm{~K}\right)$, literature & 1436 & 4989 \\
\hline Effusivity error & $<4 \%$ & $\sim 8 \%$ \\
\hline Diffusivity $\left(\mathrm{m}^{2} / \mathrm{s}\right)$ measured & $9.80 \times 10^{-7}$ & $3.25 \times 10^{-6}$ \\
\hline Diffusivity $\left(\mathrm{m}^{2} / \mathrm{s}\right)$ literature & $9.5 \times 10^{-7}$ & $3.4 \times 10^{-6}$ \\
\hline Diffusivity error & $3 \%$ & $4 \%$ \\
\hline Conductivity $(\mathrm{W} /(\mathrm{m} \mathrm{K})$, measured & 1.47 & 8.24 \\
\hline Conductivity $(\mathrm{W} /(\mathrm{m} \mathrm{K})$, literature & 1.4 & 9.2 \\
\hline Conductivity error & $5 \%$ & $10 \%$ \\
\hline $\begin{array}{l}\text { Effusivity }\left(\mathrm{J} / \mathrm{m}^{2} \mathrm{~s}^{1 / 2} \mathrm{~K}\right), \\
\text { measured including } \mathrm{R}_{\mathrm{th}}=5 \times 10^{-9} \mathrm{~m}^{2} \mathrm{~K} / \mathrm{W}\end{array}$ & 1460 & 4180 \\
\hline
\end{tabular}

lation depth of the pump laser. A telescope was used to adjust the heating laser spot size on the sample surface from $8 \mu \mathrm{m}$ to $15 \mu \mathrm{m}$. Given our limited pump power, our optimal spot size is $\sim 10 \mu \mathrm{m}$ and the corresponding optimal frequency window is between 1 and $2 \mathrm{MHz}$. The thermal effusivities of the sample A and B are summarized in Table II. The values obtained at 1 and $2 \mathrm{MHz}$ for both samples agree to within $5 \%$. This observation indicates that the $1 \mathrm{D}$ condition was satisfied. In addition, we compared the $1 \mathrm{D}$ and $3 \mathrm{D}$ solutions using the fitted thermal parameters. For this frequency window, the $1 \mathrm{D}$ and $3 \mathrm{D}$ solutions agree to within $0.5 \%$ which further validates our $1 \mathrm{D}$ analysis.

The thermal conductivities are obtained using the measured values of $e_{s}$ and $D_{s}$. A full tabulation of the measured thermal properties of samples A and B is given in Table II. While the measured conductivity for the $\mathrm{SiO}_{2}$ sample agrees closely with published values (deviation 5\%), the measured value for $\mathrm{CaF}_{2}$ only exhibit partial agreement with published values (deviation 10\%). We can think of three potential explanations for the error in our measurement. First, there may be a substantial thermal interface resistance, $R_{t h}$, between the film and substrate. Previous work by Stoner and Maris $^{29}$ considered the interface resistance between several metals and dielectrics. For a Ti film on sapphire, they measured $R_{t h} \sim 5 \times 10^{-9} \mathrm{~m}^{2} \mathrm{~K} / \mathrm{W}$ at room temperature. Using this value for the interface resistance, we find that the fitted value of $k_{f}$ and the values of $D_{s}$ for either substrate materials do not change appreciably. Because the effusivity measurement depends strongly on the boundary condition between the film and substrate, the interface resistance will more strongly influence the fitted effusivity value (listed at the bottom of Table II). Including $R_{t h}$ in our model decreases the error in the effusivity for $\mathrm{SiO}_{2}$ but increase the error for $\mathrm{CaF}_{2}$. The second source of error is related to the strong variation in the thermal conductivity of $\mathrm{CaF}_{2}$ with temperature. ${ }^{30}$ However, the temperature field does not extend far from the source. This would indicate that the effusivity measurement should be more strongly impacted because the pump and probe overlap. The third source of error stems from the fact that $\mathrm{CaF}_{2}$ is a better conductor than $\mathrm{SiO}_{2}$. Thus, the maximum 
thermal wave amplitude for any particular pump power will be larger in the $\mathrm{SiO}_{2}$ sample. Correspondingly, the SNR will be larger in the $\mathrm{SiO}_{2}$ sample leading to more accurate results. In future work, this issue will be addressed by using a more powerful pump laser to compensate for high conductivity samples. We have also considered the influence of a thin oxide layer on top of the Ti film. We performed our measurements on freshly coated samples with an oxide layer thickness of 1-2 nm. ${ }^{31}$ Our model shows that the influence of an oxide layer of this thickness does not influence the fitted results.

\section{CONCLUSION}

A photothermal reflectance technique to measure the thermal conductivity of nuclear fuel samples with high spatial resolution was developed. A thin film is applied to the sample to ensure strong optical absorption and to introduce a second boundary condition that explicitly contains an expression for the thermal effusivity. Applying the measurements in the spatial domain and frequency domain, the thermal diffusivity and effusivity of the substrate are obtained separately, and the thermal conductivity is extracted. A proof of principle experiment was conducted using two surrogate samples: $\mathrm{SiO}_{2}$ is a material with thermal properties representative of high burnup nuclear fuel, and $\mathrm{CaF}_{2}$ is a material with thermal properties representative of fresh nuclear fuel. The measured conductivities compare favorably with published values. As a post irradiation examination tool, this new experimental approach has important technological applications to advance nuclear fuel development.

\section{ACKNOWLEDGMENTS}

D.H.H. and M.K. were supported as part of the Center for Materials Science of Nuclear Fuel, an Energy Frontier Research Center funded by the U.S. Department of Energy, Office of Science, Office of Basic Energy Sciences under Award No. 2009INL-FWP1356.

${ }^{1} \mathrm{~V}$. V. Rondinella and T. Wiss, "The high burn-up structure in nuclear fuel," Mater. Today 13, 24 (2010).

${ }^{2}$ I. Cohen, B. Lustman, and J. D. Eichenberg, J. Nucl. Mater. 3, 331 (1961).
${ }^{3}$ E. Kolstad and C. Vitanza, J. Nucl. Mater. 188, 104 (1992).

${ }^{4}$ P. G. Lucuta, Hj. Matzke, and I. J. Hastings, J. Nucl. Mater. 232, 166 (1996).

${ }^{5}$ D. H. Hurley, M. Khafizov, and S. L. Shinde, J. Appl. Phys. 109, 083504 (2011).

${ }^{6}$ A. J. Schmidt, R. Cheaito, and M. Chiesa, Rev. Sci. Instrum. 80, 94901 (2009).

${ }^{7}$ M. Khafizov and D. H. Hurley, J. Appl. Phys. 110, 83525 (2011).

${ }^{8}$ A. Rosencwaig, J. Opsal, W. L. Smith, and D. L. Willenborg, Appl. Phys. Lett. 46, 1013 (1985).

${ }^{9}$ L. Pottier, Appl. Phys. Lett. 64, 1618 (1994).

${ }^{10}$ J. Hartmann, P. Voigt, and M. Reichling, J. Appl. Phys. 81, 2966 (1997).

${ }^{11}$ H. Gronbeck and M. Reichling, J. Appl. Phys. 78, 6408 (1995).

${ }^{12}$ C. A. Paddock and G. L. Eesley, J. Appl. Phys. 60, 285 (1986).

${ }^{13}$ Y. K. Koh, S. L. Singer, W. Kim, J. M. O. Zide, H. Lu, D. G. Cahill, A. Majumdar, and A. C. Gossard, J. Appl. Phys. 105, 54303 (2009).

${ }^{14}$ C. Ronchi, M. Sheindlin, D. Staicu, and M. Kinoshita, J. Nucl. Mater. 327, 58 (2004)

${ }^{15}$ P. Schoderböck, H. Klocker, L. S. Sigl, and G. Seeber, Int. J. Thermophys. 30, 599 (2009).

${ }^{16}$ F. Hemberger, H.-P. Ebert, and J. Fricke, Int. J. Thermophys. 28, 1509 (2007).

${ }^{17}$ D. G. Cahill, W. K. Ford, K. E. Goodson, G. D. Mahan, A. Majumdar, H. J. Maris, R. Merlin, and S. R. Phillpot, J. Appl. Phys. 93, 793 (2003).

${ }^{18}$ A. Mandelis, J. Appl. Phys. 78, 647 (1995).

${ }^{19}$ D. H. Hurley and K. L. Telschow, Phys. Rev. B 71, 241410 (2005).

${ }^{20}$ K. Hatori, N. Yaketoshi, T. Baba, and H. Ohta, Rev. Sci. Instrum. 76, 114901 (2005).

${ }^{21}$ W. B. Jackson, N. M. Amer, C. Boccara, and D. Fournier, Appl. Opt. 20, 1333 (1981).

${ }^{22}$ B. Li, J. P. Roger, L. Pottier, and D. Fournier, J. Appl. Phys. 86, 5314 (1999).

${ }^{23}$ G. Langer, J. Hartmann, and M. Reichling, Rev. Sci. Instrum. 68, 1510 (1997).

${ }^{24}$ R. J. Stoner and H. J. Maris, Phys. Rev. B 48, 16474 (1993).

${ }^{25}$ A. A. Maznev, J. Hartmann, and M. Reichling, J. Appl. Phys. 78, 5266 (1995).

${ }^{26}$ B. Li, L. Oittuerm, J. P. Roger, D. Fournier, and E. Welsch, Rev. Sci. Instrum. 71, 5 (2000).

${ }^{27}$ It was found that the probe beam contained high order spatial modes giving rise to unwanted structure in the phase profile. This issue was addressed by pinhole filtering the probe beam.

${ }^{28}$ D. H. Hurley, O. B. Wright, O. Matsuda, and S. L. Shinde, J. Appl. Phys. 107, 023521 (2010).

${ }^{29}$ R. J. Stoner and H. J. Maris, Phys. Rev. B 48, 16373 (1993).

${ }^{30}$ W. Hayes, M. C. K. Wiltshire, R. Berman, and P. R. W. Hudson, J. Phys. C 6, 1157 (1973).

${ }^{31}$ Initially the oxide layer on Ti is only $1-2 \mathrm{~nm}$ thick. Over several years the oxide layer can grow to $20 \mathrm{~nm}$ thick.

${ }^{32} \mathrm{CRC}$ Handbook of Chemistry and Physics, 92 nd ed., edited by W. M. Haynes (CRC Press/Taylor and Francis, Boca Raton, FL, Internet Version 2012). 